\title{
émulations
}

\section{Un esprit sain dans un corps sain.}

\section{Le yoga contemporain, une allégorie du bien-être?}

\author{
Caroline Nizard
}

Émulations - Revue de sciences sociales

2020, Varia, En ligne.

\section{Article disponible à l'adresse suivante}

https://ojs.uclouvain.be/index.php/emulations/article/view/nizard_varia

\section{Pour citer cet article}

Caroline Nizard, « Un esprit sain dans un corps sain. Le yoga contemporain, une allégorie du bien-être? », Émulations, Varia, En ligne. Mise en ligne le 6 décembre 2020. DOI : 10.14428/emulations.varia.029

Distribution électronique : Université catholique de Louvain (Belgique) : ojs.uclouvain.be

(c) Cet article est mis à disposition selon les termes de la Licence Creative Commons Attribution, Pas d'Utilisation Commerciale 4.0 International. http://creativecommons.org/licenses/by-nc/4.0/

Éditeur : Émulations - Revue de sciences sociales / Presses universitaires de Louvain https://ojs.uclouvain.be/index.php/emulations

ISSN électronique : 1784-5734

$\frac{\text { PUL PRESSES }}{\text { UNIVERSITAIRES }}$ 


\title{
Un esprit sain dans un corps sain Le yoga contemporain, une allégorie du bien- être?
}

\begin{abstract}
Caroline Nizard ${ }^{1}$
[Résumé] À partir des années 2000, le yoga connaît un succès croissant et s'est transformé pour mettre l'accent sur l'expérience corporelle, l'épanouissement personnel, éléments caractéristiques du bien-être dans le contexte sociohistorique actuel. Si les contributions en sciences sociales témoignent d'un intérêt récent pour le bien-être, rares sont les études qui se penchent spécifiquement sur les liens entre yoga et bienêtre. Grâce à des terrains ethnographiques et des entretiens menés auprès de pratiquants et de professeurs de yoga en France, en Suisse romande et en Inde, cet article interroge la manière dont les pratiquants incorporent ou non ce bien-être. Comment passe-t-on du bien-être comme obligation sociale à une intériorisation par les individus ? Partant des représentations du yoga dans les médias, cet article revient sur les raisons historiques et sociales qui ont conduit à une requalification de cette discipline. Pourtant, les réalités du terrain montrent des itinéraires de pratiques plus hétérogènes. Selon l'implication dans la pratique du yoga moderne, les glaneurs mettent l'accent sur l'épanouissement dans le bien-être, alors que ceux qui s'astreignent le plus à une maîtrise de leur corps, de leurs actions (les enthousiastes et les passionnés) valorisent plutôt la santé et/ou la spiritualité.
\end{abstract}

Mots clés : Bien-être, injonction sociale, yoga, autonomie, anthropologie.

[Abstract] Since the 2000s, yoga has become very popular and has shifted its focus to the body experience and personal development, characteristic features of wellbeing. While social sciences highlight a recent interest in wellbeing, there are few empirical researches that focus on the links between yoga and wellbeing. Using ethnographic fieldwork and interviews with yoga practitioners and teachers in France, French-speaking Switzerland and India, this article examines how practitioners incorporate or not this wellbeing. How do we move from wellbeing as a social obligation to its individual internalization? The article focuses on the representations of yoga in the media, and on the historical and social reasons that have contributed to the recharacterization of this practice. However, ground realities show that more there are heterogeneous practice trajectories. According to their involvement in the practice of modern yoga, some practitioners (the gleaners) highlight wellbeing, whereas those who are most committed to controlling their bodies and their actions (the enthusiasts and passionate practitioners) emphasize other dimensions of yoga, such as health and/or spirituality.

Keywords: Wellbeing, social pressure, yoga, autonomy, anthropology.

\footnotetext{
${ }^{1}$ Université de Lausanne, IHAR/THEMA, Suisse.
} 


\section{Introduction}

Tirée des Satires de Juvénal (datées entre 90 et 125 ap. J.-C.), l'expression Mens sana in corpore sano enjoint les hommes à cesser d'implorer vainement les dieux et à s'interroger sur ce qui importe vraiment : la santé physique et mentale. De nos jours, si cette aspiration perdure, elle ne s'adresse plus aux dieux, mais à l'homme qui doit trouver les moyens nécessaires pour accéder à l'harmonie entre « corps et esprit ». De ce principe est né un impératif : "prendre soin de soi » (Foucault, 1984). Michel Bernard note qu'entre les années 1970 et aujourd'hui, le droit de posséder un corps et d'en jouir s'est transformé d'abord en « droit au bien-être » (Bernard, 1976:13), puis en devoir de prendre soin de son corps, de s'imposer une hygiène de vie. Depuis l'ouvrage de Carl Cedeström et André Spicer, (2016), les quotidiens titrent régulièrement sur «l’injonction du bien-être $»^{2}$, «l'obsession $»^{3}$ du bonheur, où le yoga apparaît comme l'un des moyens pour y parvenir.

Dans le langage courant, le bien-être est compris comme un « état agréable résultant de la satisfaction des besoins du corps et du calme de l'esprit » (Larousse). Cet état renvoie à des sensations, des émotions ou des réponses à des besoins physiques, mentaux, voire émotionnels. Selon Olivier Moco (2007), ce terme apparaît la première fois sous la plume d'Alexis de Tocqueville, qui crée un lien avec le politique. Auparavant associée au bonheur, aujourd'hui, cette notion est souvent synonyme de santé. Dans le préambule de 1946 de l'OMS, la santé y est définie comme « un état de complet bien-être physique, mental et social, [qui] ne consiste pas seulement en une absence de maladie ou d'infirmité $»^{4}$.

Pour Alain Ehrenberg 5 , le bien-être est une notion large et polymorphe. C'est pourquoi, il préfère n'en donner que des caractéristiques : cet état s'inscrit (a) dans une expérience subjective, (b) centrée sur l'individu, (c) les sensations, l'intériorisation, et (d) oscille entre santé et détente. Par ailleurs, (e) il ne peut être compris sans être replacé dans le contexte sociohistorique actuel (voir infra). Selon Nadia Garnoussi, dans nos sociétés, la «multiplication des références au bien-être exprime plutôt le façonnage d'un idéal existentiel, pragmatique, utilitariste, où l'épanouissement individuel

\footnotetext{
2 URL : https://www.lemonde.fr/m-perso/article/2018/08/28/eva-illouz-l-injonction-au-bonheur-est-une-trouvaille-formidable-pour-le-pouvoir_5346894_4497916.html. Consulté le 23.07.19.

${ }^{3}$ https://www.lexpress.fr/styles/forme/quand-la-quete-du-bien-etre-tourne-a-l-obsession_1793690.html. Consulté le 23.07.19.

4 Cette définition provient du préambule de la constitution de I'OMS. URL: https://www.who.int/about/who-we-are/frequently-asked-questions. Consulté le 12.04.18.

5 Conférence «Bien-être, santé mentale, autonomie » donnée par Ehrenberg lors du colloque Bien-être au Nord et au Sud: explorations, contradictions, pouvoir et pratiques du 22 au 25 octobre 2019, organisé par Sciences Po de l'Université de Bordeaux.
} 
constitue avant tout un état qui se construit rationnellement, s'obtient et s'évalue $»^{6}$. Certaines pratiques dites de développement personnel et/ou les méthodes psychocorporelles, comme le yoga ou la méditation, mettent l'accent sur les sensations, l'expérience corporelle, et deviennent ainsi des méthodes favorables pour développer ce bien-être (Heilbrunn, 2019).

Cet article souhaite comprendre s'il existe ou non un processus de requalification du yoga en tant que méthode de bien-être dans les médias et parmi les pratiquants de yoga. Il vise aussi à comprendre comment les pratiquants de yoga intériorisent cette injonction au bien-être et quelle importance le bien-être occupe selon les itinéraires de pratiques.

\section{Regards anthropologiques sur le bien-être dans le yoga}

Le terme yoga vient du sanskrit yug, qui signifie « union », «lien» ou « joint ». Selon James Mallinson et Mark Singleton (2017), il a connu un panel d'interprétations et de réappropriations au cours de l'histoire, le faisant osciller entre un but (pour sortir du cycle des réincarnations chez les hindous) et un moyen (doté d'une palette de techniques). Ici, le yoga sera compris comme une méthode psychocorporelle qui mobilise des exercices physiques et mentaux teintés, au gré des choix, d'ascétisme physique, de santé, de bien-être et/ou d'ésotérisme (Hauser, 2013)7. Au cours de l'histoire, trois dimensions du yoga - la spiritualité, la santé, le corporel - prédominent différemment, se complètent, se confrontent ou se reconfigurent (Nizard, 2019). L'une des spécificités du yoga apparaît justement dans ces usages pluriels où chaque pratiquant peut enchevêtrer ces dimensions, en valoriser certaines et y puiser des outils, en fonction de sa motivation, de ses questionnements et de son implication.

Si les travaux des historiens (Alter, 2004 ; De Michelis, 2005 ; White, 2012), des sanskritistes (Mallinson, Singleton, 2017; Singleton, 2010) ou des sociologues (Altglas, 2005; Hoyez, 2012) se multiplient sur le yoga, les imaginaires autour du bien-être restent quasiment inexplorés parmi les spécialistes du yoga, tandis que les représentations populaires sur ces liens abondent (Ali-Knight, Ensor, 2017). Historiquement, les effets positifs du yoga sur la santé figurent déjà dans des textes anciens (comme le Hatha Yoga Pradipika, $\mathrm{xv}^{\mathrm{e}}$ siècle), mais il faut attendre les années 1920, en Inde, pour voir naître de véritables recherches ayant pour vocation de démontrer ces bienfaits. Depuis une vingtaine d'années, plusieurs auteurs décrivent une amplification de l'intérêt pour la

\footnotetext{
${ }^{6}$ Conférence « La recherche du bien-être » donnée par Nadia Garnoussi à l'EHESS lors de la présentation de l'ouvrage paru en 2008 d'l. Coutant et S. Wang (dir.), Santé mentale et souffrance, le 12 décembre 2019, organisée par l'EHESS. En ligne, consulté le 14.04.20 URL: http://iris.ehess.fr/index.php?4196.

7 “ The term yoga has been associated with various distinctive blends of mental and physical exercises performed in order to achieve some sort of improvement - or even perfection whether conceived in terms of esotericism, fitness, self-actualization, body aesthetics, or healthcare." (Hauser, $2013: 2$ )
} 
dimension sanitaire/thérapeutique dans les pratiques de yoga (Hauser, 2013 ; Strauss, 2005) en Inde, aux États-Unis ou en Europe.

En Inde, comme aux États-Unis, les études médicales foisonnent sur ce sujet et ont souvent pour objectif d'évaluer l'impact des postures, de la méditation (Dinesh, 2013; Jerath et al., 2012) ou des techniques de respiration sur le cerveau (Cameron, 2001; Price, Hooven, 2018), la pression artérielle (Prakash, Upadhyay-Dhungel, 2015), la concentration (Saraswati, 2009) et sur certaines pathologies (diabète, asthme, maladies cardio-vasculaires, notamment), laissant de côté les discours. Sur l'ensemble de ces études, les plus abondantes portent sur les effets positifs de la pratique des postures de yoga ou de la méditation (Brown, Ryan, 2003) sur la santé. Ici, la santé est comprise dans son versant thérapeutique, curatif, bien plus que préventif ou rattaché au bienêtre.

Hors du champ spécifique du yoga, la question du bien-être occupe un nombre croissant de recherches dans de nombreuses disciplines. Les psychologues travaillent sur la qualité de vie (par exemple, Alexandrova, 2017) ou sur le développement de la psychologie positive (Seligman, 2013), tandis que les économistes s'efforcent de déterminer les facteurs de bien-être et leur impact sur l'économie et le monde de l'entreprise (Frey, 2011 ; Schulte et al., 2015). Dans Culture and Well-Being (2008), Corsín Jiménez montre combien les définitions et les caractéristiques du bien-être varient selon les contextes socioculturels et répondent aux exigences de sociétés néolibérales et globalisées.

Rémy Pawin (2014) a retracé l'épistémologie de la notion et souligne que ce champ de recherche est apparu bien plus tardivement dans les sciences sociales en France (vers les années 1970) qu'en Angleterre (1912), car elle a longtemps été considérée comme peu « légitime » (Pawin, 2014 : 286). Aujourd'hui s'opposent des travaux souhaitant mesurer, voire objectiver le bien-être, et des recherches sur les perceptions subjectives. Ces dix dernières années, les sociologues (Cabanas, Illouz, 2018; Cederström, Spicer, 2016; Heilbrunn, 2019) s'intéressent spécifiquement à la question du bien-être (Forsé, Langlois, 2014) ou à cette modalité dans d'autres pratiques psychocorporelles (Héas, 2020 ; Perrin, 1984) ou en lien avec la santé (Garnoussi, 2018). Cette réflexion se positionne dans le sillon des travaux d'Ehrenberg (2011), de Cederström et Spicer, (2016), de Cabanas et Illouz, (2018), de Garnoussi (2018) qui s'accordent pour allier modernité, responsabilité individuelle et intérêt accru pour le bien-être.

\section{Contexte et caractéristiques sociales des pratiquants de yoga}

Selon Ysé Tardan-Masquelier (2002), en Europe, le yoga s'est progressivement détaché de ses fondements philosophiques et spirituels pour mobiliser des arguments plutôt en faveur de la santé et du bien-être. Plusieurs auteurs (Hauser, 2013; Newcombe, 2005) ont apporté des nuances à cette interprétation, même s'il reste indéniable que le yoga a gagné en popularité grâce à (ou à cause de) cette association avec le bien-être. 
Des travaux sur l'histoire de la diffusion du yoga en France (Ceccomori, 2001) et en Suisse (Desponds Meylan, 2007) ont mis au jour des transformations et des moments de rupture depuis l'importation du yoga. Schématiquement, en France, le yoga naît en 1895 de la conjonction de deux mouvements : l'exportation par des réformateurs hindous tels que Vivekananda ${ }^{8}$ et l'importation par les orientalistes, la Société théosophique $^{9}$ et les sanskritistes français, qui vouent une fascination à l'Inde. Importé comme un système philosophique et une spiritualité, le yoga s'adresse d'abord à une élite intellectuelle.

À partir des années 1940-1950, aux dimensions philosophiques se mêlent des exercices de postures et de respiration. Les premiers enseignants de yoga européens se forment en Inde avant de fonder leurs écoles en France ou en Suisse. La plupart souhaite délivrer un enseignement dans la lignée de ceux qui sont reconnus comme des gourous $^{10}$ dans les milieux du yoga. Il faut attendre les années 1960-1970 et des transformations sociales liées à Mai 68 pour que cette pratique trouve son public dans la contre-culture. Il se rattache alors à l'image d'une pratique « New Age », où des célébrités, comme les Beatles, participent à ces représentations et à l'ouverture vers un public plus large. Ainsi, cette discipline connaît de nouvelles transformations, puisque les professeurs européens élaborent des mélanges entre philosophie, spiritualité et d'autres techniques corporelles ou de relaxation. Les enseignants simplifient les aspects philosophiques et les notions liées à l'hindouisme en insistant plutôt sur les découvertes scientifiques qui viennent attester les bienfaits du yoga sur la santé. Ces profonds bouleversements marquent l'émergence de nouveaux mouvements religieux, notamment au sein de certains courants de yoga (Altglas, 2005).

Silvia Ceccomori (2001) et Anne-Cécile Hoyez (2012) soulignent qu'au début des années 1980 , on assiste à une perte d'intérêt pour le yoga par le public européen ${ }^{11}$. Au contraire, depuis les années 2000, la multiplication de courants de yoga hybrides venus

\footnotetext{
${ }^{8}$ Vivekananda (1863-1902) est un penseur, réformateur indien qui a grandement participé à la diffusion du yoga dans le monde, notamment au Parlement mondial des religions à Chicago en 1893. II présente un hindouisme rénové, teinté de réformes sociales et morales, un modèle de tolérance et de pragmatisme.

${ }^{9}$ Basée sur une rhétorique du dépassement des cadres chrétiens, la Société théosophique peut être comprise comme une société d'initiés passionnés de spiritisme et d'orientalisme qui effectue des mélanges syncrétiques entre le christianisme et les philosophies orientales, notamment indiennes. Elle a joué un rôle clef dans la réception des traditions indiennes dans le monde.

${ }^{10}$ Issu du sanskrit, le terme « gourou » se compose de « gu », ignorance et « ru », dissiper. En Inde, le gourou désigne un maître, un guide spirituel et ne revêt pas de connotation péjorative.

${ }^{11}$ Cette perte d'intérêt est difficile à mesurer étant donné qu'il n'existait pas de statistiques précises sur cette pratique. Les raisons évoquées par les auteurs seraient liées à des conflits suite à la création de plusieurs fédérations de yoga à partir de 1965 et à la fermeture de nombreux cours de yoga non rattachés à ces fédérations.
} 
des États-Unis donne à voir à la fois une occidentalisation du mouvement et une autonomisation de la transmission du yoga par rapport à l'Inde. Mark Singleton (2010) et Elisabeth De Michelis (2005) démontrent que plusieurs courants de yoga naissent aux États-Unis (comme le Bikram yoga, le Power yoga, le Hot Yoga, le courant Jivamukti). Ces derniers se détachent souvent de l'Inde, de l'hindouisme et prônent une « adaptation du yoga au corps des Européens » en mettant l'accent sur l'activité physique, le bien-être et/ou une spiritualité. Généralement, ces courants se cristallisent autour de pratiques identifiables, tout en apportant une innovation ${ }^{12}$. Chaque courant s'accompagne de la création de formations courtes de professeurs, de niches commerciales (Mori et Squarcini, 2008), d'un tourisme du bien-être (Lehto et al. 2006). La pratique s'apparente désormais sous certains aspects au sport, au bien-être et à la santé. Ces transformations du yoga résultent de changements dans les publics intéressés par le yoga, dans les finalités de la pratique (spiritualité, santé, bien-être et/ou sport) et de l'évolution du contexte sociohistorique, comme nous allons le voir autour du bien-être.

Selon une enquête sur les activités physiques et sportives menée par l'INJEP' 13 , 1,9\% de la population française pratique le yoga en 2010. En 1997, le yoga est apparu pour la première fois dans la nomenclature des enquêtes de l'INSEE. À cette époque, il était comptabilisé avec la danse, la gymnastique, le Tai Chi Chuan. Aussi, il est difficile d'évaluer l'augmentation du nombre de pratiquants. En France, le journal spécialisé Esprit yoga estime à 1,4 million de pratiquants en 2014 et à 2,6 millions en 2019. Une autre piste consiste à recenser le nombre de professeurs de yoga. Pourtant, si la première fédération de yoga (la Fédération française d'Hatha Yoga) est apparue en 1966, les professeurs n'ont rapidement plus eu besoin d'être rattachés à une fédération pour exercer (Ceccomori, 2001). Aujourd'hui, de nombreux professeurs enseignent sans appartenir à une fédération. Une autre piste pour montrer l'ampleur du phénomène est la croissance du nombre de vidéos sur Internet. À la date du 23 juillet 2014, Google recensait 3540000 vidéos et au 12 septembre 2018 environ 23600000 de vidéos, mettant en scène une pratique personnelle où un professeur explique une posture ou donne un cours ${ }^{14}$. Ces chiffres doivent être pris avec beaucoup de précautions, tant que la prochaine enquête sur les pratiques physiques et sportives prévue en mai 2021 ne sera pas publiée et ne donnera pas une image plus fiable de ces transformations. Ces quelques

\footnotetext{
${ }^{12}$ Par exemple, le Bikram yoga propose des cours dans des salles chauffées à $40^{\circ}$, et le courant de la Kundalini met l'accent sur des mouvements très répétitifs permettant de " faire circuler l'énergie».

${ }^{13}$ Enquête sur les Pratiques physiques et sportives (EPPS) - 2010, CNDS, INSEP, MEOS - ministère des Sports, publiée en 2016. En ligne, consulté le 22 juillet 2019. URL :https://www.epsilon.insee.fr/jspui/bitstream/1/63075/1/INJEP_horscollection_1_2016.pdf.

${ }^{14}$ Internet, les applications entraînent de nouveaux modes d'apprentissage dans de nombreux domaines. Ainsi, cette augmentation du nombre de vidéos n'est pas l'apanage du yoga.
} 
exemples illustrent quoi qu'il en soit une expansion rapide et une commercialisation grandissante autour du yoga ${ }^{15}$.

Cette étude ${ }^{16}$ se fonde sur une approche anthropologique qui combine observation participante et entretiens. Entre 2013 et 2017, soixante entretiens semi-directifs ont été réalisés auprès de pratiquants et/ou de professeurs de yoga ${ }^{17}$ à Paris (23), en Suisse romande (22, Gruyère et Lausanne) et en Inde (15, à Bangalore, Mysore, Neyyar Dam, Delhi, Rishikesh). Parmi ces entretiens, une trentaine ont fait l'objet d'un suivi longitudinal ${ }^{18}$.

L'observation participante s'est déroulée lors de cours de yoga hebdomadaires pendant cinq ans en Suisse, en France et plus ponctuellement en Inde, et lors de différents événements : festivals de yoga (à Rishikesh), conférences (Congrès européens de yoga à Zinal, en Suisse) et retraites en ashrams (Sivananda à Orléans, en Ardèche et en Inde du Sud) pendant une semaine à un mois (se reporter à l'annexe). Comme de nombreux pratiquants naviguent entre les professeurs, j'ai choisi de suivre des professeurs affiliés à sept courants de yoga afin d'observer des cours plus portés sur la dimension physique (ashtanga, vinyasa), thérapeutique (Iyengar, nidra) et/ou spirituelle (Sivananda, kundalini, de Shribhashyam). Les professeurs se reconnaissent autour du terme générique d'Hatha yoga qui embrasse cette diversité (Singleton, 2010). Enfin, si les dimensions sociologiques du yoga ont fait l'objet de nombreux travaux dans les pays anglophones (De Michelis, 2005 ; Newcombe, 2005 ; Singleton, 2010), Béatrix Hauser (2013) souligne

\footnotetext{
${ }^{15}$ Singleton (2010) parle d'un marché autour du yoga de 5,7 milliards de dollars en 2008 aux États-Unis, Ruiz (2010) évoque un marché mondial annuel de 18 milliards de dollars et AliKnight et Ensort (2017), de 16 milliards de dollars en 2012. C'est un marché difficile à évaluer en particulier en France, les études INSEE évaluaient le marché du bien-être à 37,5 millions d'euros en 2015. URL: https://www.insee.fr/fr/statistiques/4238423?sommaire=4238781. Consulté le 17 avril 2020.

${ }^{16}$ Les éléments de terrain présentés ici s'inscrivent dans une recherche plus générale dont l'objectif était de comprendre comment et pourquoi, par l'apprentissage du yoga, les pratiquants changent ou non leur rapport à leur corps, au monde et ses conséquences sur leur mode de vie (relations sociales, pratiques alimentaires, médicales, etc.).

${ }^{17}$ Caractéristiques sociales des soixante enquêtés :

Professeur de yoga (15), ayant fait une formation de professeur sans l'enseigner (14), pratiquant n'ayant pas fait de formation (31).

Sexe : Hommes (20), Femmes (40);

Âge le jour de l'entretien : - de 30 ans (4), 30 à 39 ans (22), 40 à 49 ans (19), 50 ans et plus (15) ;

Nombre d'années de pratique : - de 5 ans (20), 5 à 10 ans (13), 10 à 15 ans (17), supérieur à 15 ans (10);

Catégories socioprofessionnelles : cadres (24), professions intermédiaires (13), employés (6), indépendants, chefs d'entreprise (12), inactifs (2), retraités (3).

${ }^{18}$ Inscrire les parcours de vie tout au long des terrains était un choix afin d'observer les changements de comportements déclarés et observés notamment sur les réseaux sociaux.
} 
que les recherches sur le yoga dans les contextes non anglophones restent moins abondantes. Ce constat a motivé le choix des terrains en France et en Suisse.

Comme les pratiques de gymnastique, de fitness, l'enquête de l'INJEP montre que le yoga est une pratique genrée, majoritairement féminine (Eisenmann, 2019; Hoyez, 2012 ; Newcombe, 2005). À la lumière de mes terrains, en France, deux tiers des pratiquants rencontrés et les trois quarts en Suisse sont des femmes. Le yoga touche généralement les 30-55 ans avec un âge moyen de 41 ans. Les classes sociales moyennes et surtout supérieures sont prédominantes parmi les personnes rencontrées, et plus spécifiquement dans les domaines médicaux ou paramédicaux, et sociaux ${ }^{19}$. L'enquête de l'INJEP observe que le yoga est prisé par les cadres, ceux qui ont suivi des études supérieures ou les urbains. Ces pratiquants présentent donc une forte homogénéité sociale.

\section{Représentations du bien-être dans les journaux spécialisés du yoga}

L'enquête de l'INJEP souligne que la santé et le bien-être, assimilé dans ce contexte à la détente, sont les motifs principaux évoqués par les pratiquants de yoga pour pratiquer un sport. Dans un autre registre, l'analyse des discours dans les journaux spécialisés permet de rendre compte des représentations sociales qui gravitent autour de ces pratiques et de montrer comment le bien-être a gagné en importance au point de changer les finalités et les définitions du yoga. De nos jours, on peut trouver trois magazines grand public dédiés au yoga dans les kiosques français : Esprit Yoga, Énergie, équilibre et bien-être; Yoga Journal et Yoga Magazine ${ }^{20}$. Ces journaux illustrent bien l'évolution de l'usage du yoga dans cette perspective de bien-être. L'analyse a été réalisée à partir de cinquante-six numéros de trois revues commercialisées dans les kiosques en France et publiées entre janvier 2014 et décembre 2017 : Esprit Yoga (24 numéros), Yoga Journal (8) ${ }^{21}$ et Yoga Magazine (24). Ces données restent insuffisantes pour généraliser tout propos. Pour autant, les travaux de Mimi Nichter (2000), de Nandini Bhalla et Leigh

\footnotetext{
${ }^{19} 24$ personnes travaillent dans le domaine médical/paramédical, 12 dans le social. Cette forte proportion de personnes travaillant dans les milieux du social et médico-social se retrouve dans d'autres recherches sur le développement personnel (Marquis, 2014) ou d'autres pratiques de «spiritualité holiste » (Sointu, 2006).

${ }^{20}$ Les journaux sur le yoga existent depuis les années 1960. Initialement destinés à un public de spécialistes et de professeurs de yoga, les journaux se sont ensuite diffusés bien au-delà de la sphère professionnelle dans les années 2000. Le premier a été édité la première fois au printemps 2011, le second en mars 2015 et le dernier en juin-juillet 2015. J'ai aussi comparé ces journaux aux Cahiers du Yoga publiés par l'association Yoga 7 en Suisse. Ce journal édité tous les quatre mois environ n'est pas disponible dans les kiosques. II faut soit être abonné, soit acheter le magazine sur le site. II s'adresse uniquement aux enseignants de yoga et met l'accent sur la philosophie et la spiritualité.

${ }^{21}$ Le premier numéro date de mars 2015.
} 
Moscowitz (2019), de Liina Puustinen et Matti Rautaniemi (2015) renforceront certaines observations. Si les deux premiers portent sur les magazines de yoga américains, Puustinen et Rautaniemi (2015) ont réalisé une analyse plus générale sur les journaux féminins finlandais, et certains dédiés au yoga. Ils soulignent que, dans les magazines, le yoga est d'abord associé à une pratique permettant de trouver un équilibre holistique entre le corps et l'esprit, et que les journaux non spécialisés mettent l'accent sur la santé, le bien-être, la réduction du stress et dans un second temps sur la spiritualité.

Esprit Yoga, Yoga Journal et Yoga Magazine sont structurés en plusieurs rubriques et s'adressent principalement aux femmes. Par exemple, sur les cinquante-six numéros du corpus, quarante-neuf couvertures représentent des femmes. Sur les quinze contributeurs d'Esprit Yoga, douze sont des femmes. Dans Yoga Journal, les contributeurs changent régulièrement, mais les journalistes restent le plus souvent des femmes. D'ailleurs, dans les kiosques, ces journaux sont vendus sous la rubrique « magazines féminins » et non « sport ». Pour prendre un exemple représentatif, sur les cinquante-neuf photos dans le numéro 46 d’Esprit Yoga (nov.-déc. 2018), quarante-neuf exposent des femmes. Elles sont toutes blanches, minces, voire très minces. Nichter (2000) ainsi que Bhalla et Moscowitz (2019) soulignent que les magazines promeuvent des images de femmes minces, jeunes, blanches et hétérosexuelles ${ }^{22}$.

La femme pratiquant le yoga n’est pas forcément une passionnée, car les postures présentées peuvent être de niveau débutant ou plus avancé. Cette presse spécialisée s'adresse à la femme de la classe moyenne ou supérieure qui travaille, qui a des enfants et souhaite pratiquer en famille, ce qui renvoie aux caractéristiques sociales de l'enquête de l'INJEP. Cette pratiquante a aussi certaines pratiques de consommation: elle nourrirait un intérêt à l'égard de l'écologie, tout d'abord parce qu'elle serait végétarienne. L'ensemble des cinquante-six rubriques « cuisine » des trois magazines offre des recettes végétariennes ou végétaliennes et donne des conseils pour devenir végétarien ou du moins flexitarien ${ }^{23}$. Par exemple, Esprit Yoga a une rubrique destinée à la nutrition et explique " comment devenir végétarien », " manger cru », " sans gluten », " cultiver son jardin bio ». Suite au succès d'Esprit Yoga, son éditeur Andrea Semprini a créé un second journal Esprit Veggie, cuisine et art de vivre végétarien, où les abonnés du premier bénéficient de réduction pour le second. Ce soin à son corps se traduit aussi par un intérêt certain pour les thérapies non médicamenteuses comme le reiki, l'hypnose, les fleurs de Bach et l'aromathérapie. Les conseils vont jusqu'aux expositions à voir, à la musique à écouter (chants de mantras) ou aux lectures (principalement des livres de yoga en poche ou de développement personnel). Luca Mori et Federico Squarcini

\footnotetext{
22 « By tracing the evolution of magazine covers in the journal, this research has shown the ways in which the practice has become associated with mainly white women, slim and toned bodies, vegetarianism or veganism, upper to middle-class lifestyle, commercialization, and mysticism, a supernatural spirituality » (2019: 94).

${ }^{23}$ Terme qui désigne les personnes qui mangent occasionnellement de la viande et du poisson.
} 
remarquaient déjà ces mêmes caractéristiques en 2008 autour de la commercialisation du yoga.

Dans une étude qualitative sur l'évolution des représentations du yoga dans les journaux spécialisés de yoga aux États-Unis entre 1975 et 2016, Bhalla et Moscowitz (2019) montrent que si dans les années 1975, le yoga est d'abord valorisé comme une pratique spirituelle, aujourd'hui l'accent porte sur le bien-être, le développement personnel et la commercialisation. Dans ces journaux, le bien-être est compris comme une "détente ", un « apaisement du mental » et permettrait de développer «l'autonomie », « la flexibilité », « le lâcher-prise ». C’est par exemple le cas d'un article d'Esprit Yoga, «Éloge de la souplesse $»^{24}$. Nathalie Ferron ${ }^{25}$ part de l'idée que l'individu est soumis à des pressions (notamment professionnelles et familiales). Cet article élogieux du yoga illustre une pratique «bonne pour la santé, la socialisation, le travail » et un instrument «idéal de lutte contre le stress ». Les risques encourus par celui qui ne le pratiquerait pas sont « la maladie, la solitude, la perte, la dépression ». Certaines valeurs sociales contemporaines influencent le discours autour du yoga puisque le bien-être se baserait sur une " démarche individuelle », de "connaissance », de "quête et de maîtrise de soi ». Ces journaux, comme dans cet exemple, mobilisent un vocable autour de la flexibilité et de la performance. Or, Cederström et Spicer (2016) montrent combien les discours sur le développement personnel empruntent toujours plus au champ lexical de l'entreprise. Ils étudient la manière dont les sociétés modernes amènent les individus à être responsables de ces changements et ces « injonctions au bien-être ».

\section{Comprendre la place du bien-être chez les pratiquants rencontrés}

Les quelques journaux pris en compte illustrent pourquoi yoga et bien-être semblent être associés. Pourtant, dans les discours des pratiquants rencontrés, le bien-être est souvent présenté comme une modalité secondaire après la dimension thérapeutique, la spiritualité (Nizard, 2020d) ou la philosophie. Les pratiquants définissent le bien-être comme une sensation "de détente», une harmonie entre "corps et esprit ». Amélie ${ }^{26}$ (pratiquante, Française, 38 ans) ressent de la « joie», Jade (professeure, Suisse, 44 ans) parle de «paix », Antoine (pratiquant, Suisse, $41 \mathrm{ans)}$ évoque une « harmonie entre le corps et l'esprit » ou encore le fait d'être « recentré » sur sa "vérité intérieure ». Beaucoup de personnes témoignent du sentiment d'être " apaisé », « détaché ». Ce vocabulaire montre une construction du sentiment par une mise en adéquation entre l'idée du « bien-être », la sensation de « se sentir détendu » et un vocable propre à la discipline

\footnotetext{
${ }^{24}$ N. Ferron, 2013, «Éloge de la souplesse », Esprit Yoga, n 12, p. 24-27.

${ }^{25} \mathrm{~N}$. Ferron est journaliste spécialiste des thématiques de santé, psychologie, spiritualités orientales et professeure de yoga.

${ }^{26}$ Les prénoms ont été anonymisés, mais pas les caractéristiques sociales.
} 
comme « se sentir recentré » ou " être dans le moment présent », lexique fréquemment employé dans les cours de yoga. Dans les discours, le bien-être n'est pas forcément associé à la santé qui est plutôt comprise dans son versant thérapeutique, curatif ou pour soulager des douleurs.

Dans les motivations premières, chez les Français et les Suisses rencontrés ${ }^{27}$, ce sont des problèmes de santé importants psychiques (dépression, burn out) ou physiques (cancer, ou des maladies chroniques) qui les ont décidés à débuter le yoga. Les enquêtés évoquent aussi une période de bouleversement dans leur vie (perte d'emploi, déménagement, deuil d'un proche) qui entraîne souvent de nombreux changements de comportement. Ces deux motifs marquent une rupture qualifiée de « bifurcation ${ }^{28}$ (Grossetti, Bessin, Bidart, 2009), à l'origine d'une remise en question profonde et l'adoption de nouveaux modes de vie. Parmi les autres causes, les personnes rencontrées témoignent de stress important dans leur vie personnelle et/ou professionnelle. Dans une moindre mesure, les enquêtés disent avoir découvert le yoga "par hasard ", "par ennui », lors d'un voyage ou par leur entourage. La spiritualité comme le bien-être apparaissent peu comme motivation première. Ces personnes évoquent plutôt des raisons liées de près ou de loin à la santé ou à l'adaptation aux contraintes de la vie contemporaine.

Alors que le bien-être est rarement une motivation pour débuter, il reste un leitmotiv dans les entretiens. Il occupe une place à géométrie variable selon l'implication dans la pratique. Il existe plusieurs itinéraires possibles de pratiques, synthétisés imparfaitement par une typologie. La typologie ${ }^{29}$ présentée ici est le résultat de l'analyse des

${ }^{27}$ Santé : 18 ; bouleversement : 9 ; stress : 7 ; hasard, ennui, voyages : 5 ; bien-être : 3 ; spiritualité $: 2$; entourage : 1 . Ces chiffres concernent les pratiquants rencontrés et n'ont pas vocation à être généralisés.

${ }^{28}$ La notion de bifurcation se caractérise par (a) une rupture forte avec un « avant » et un " après », (b) la prise de conscience de l'événement, (c) la redéfinition de soi, (d) les pratiques ascétiques et les changements profonds dans les modes de vie (Grossetti et Bidart, 2009).

${ }^{29}$ La typologie de l'implication résulte de l'analyse des discours et du croisement entre (a) la définition donnée du yoga plutôt comme un loisir, une boîte à outils ou un mode de vie, (b) les motivations de départ, (c) l'importance du yoga dans la vie quotidienne (fréquence de la pratique, l'introduction d'une pratique individuelle chez soi par exemple), (d) l'adhésion à des normes et des valeurs, (e) le nombre de domaines (alimentation, pratique médicale, relation aux autres, travail) impactés depuis le début du yoga, (f) ainsi que certaines étapes du processus ayant permis des basculements d'une catégorie à l'autre (les séjours en ashram, la formation de professeur). Pour autant cette typologie ne répond pas à la construction des types-idéaux de Weber (1992) puisqu'il ne s'agit pas de traiter de grands statuts sociaux, mais de dégager des implications et des processus de changements. Cette typologie a été construite grâce aux données empiriques, et ont été remises en question grâce à l'analyse et la comparaison des données. De plus, elles ne cherchent pas à décrire toute la réalité sociale, mais seulement à mettre au jour des réseaux d'actions autour des pratiquants rencontrés. Enfin, ces typologies apparaissent beaucoup plus flexibles puisqu'elles reflètent des modalités d'actions possibles pour un pratiquant à un moment donné. 
données de terrain et renvoie plutôt à une typologie d'actions que de personnes, puisqu'un même individu peut selon sa pratique, ses aspirations ou son parcours de vie passer d'un statut à un autre (Nizard, 2019). Cette typologie ${ }^{30}$ de l'implication (glaneur, enthousiaste, passionné31) s'avère pertinente analytiquement pour rendre saillants des itinéraires possibles de transformations chez les personnes rencontrées et comparer les actions des pratiquants dans les modes de vie. Ici, elle permet aussi de montrer des différences de conception du bien-être. Par ailleurs, un même pratiquant peut, au cours de son apprentissage, de son parcours et de ses choix, changer de catégorie, voire ne plus y appartenir du tout.

Par exemple, pour Dorothée, l'une des principales raisons pour continuer c'est «ce sentiment de bien-être après le cours. Pendant quelques minutes, parfois mêmes quelques heures, j'ai l'impression que rien ne pourrait m'atteindre, comme si j'étais dans une bulle. Je vois les choses de l'extérieur » (Dorothée, pratiquante, 40 ans). Si tous les pratiquants recherchent cette détente, d'autres dimensions prédominent, comme en témoigne Delphine.

«Je me souviendrai toute ma vie de mon premier cours, je me suis sentie planer complètement. [...] C'est un état auquel j'aspire toujours et que je ne retrouve pas à chaque fois. [...] Pour moi, le yoga c'est un outil qui me permet de mieux gérer mes émotions, de prendre conscience de mon alimentation, d'être à l'écoute de mon corps et de mes besoins. [...] II me permet de révéler la personne que je suis vraiment. » (Delphine, pratiquante, 38 ans)

Le discours de Delphine sous-entend des changements de comportements qui dépassent le seul bien-être. Enfin, d'autres pratiquants comme Amandine critiquent ces usages du yoga.

«Je crois vraiment qu'il faut avoir une discipline. Le feel good yoga, avec "aujourd'hui j'ai pas trop envie de faire du yoga parce qu'il fait beau" ou "j'ai envie aujourd'hui pour me sentir bien", ce n'est pas du yoga. À ce moment-là, on va faire de la gym douce, du Pilates, de la relaxation. Je crois que dans le yoga il est indispensable d'avoir un engagement, une régularité. » (Amandine, professeure, 30 ans, Suisse)

Trois profils et trois définitions du yoga se distinguent : les glaneurs, pour qui le yoga est une « boîte à outils » permettant de répondre aux maux quotidiens et dont la pratique varie en fonction des besoins (comme Dorothée), les enthousiastes, pour qui le yoga implique d'agir sur eux-mêmes et qui témoignent de la mise en place de changements dans plusieurs domaines de leur vie (comme Delphine), les passionnés, pour qui le yoga devient un véritable mode de vie, une hygiène nécessitant à leurs yeux,

30 ॥l s'agit plutôt de profils qui mettent en évidence certaines étapes et comportements, qu'une construction de types-idéaux weberiens.

${ }^{31}$ Les noms donnés aux différents profils (glaneur, enthousiaste et passionné) ne proviennent ni du langage émique, puisque les pratiquants ne se définissent pas ainsi (même si certains se disent « passionnés par le yoga »), ni de la littérature scientifique. 
exercices, répétitions, voire ascèse (comme Amandine). À travers ces discours, les aspirations, les finalités et définitions du bien-être sont variées et illustrent autant d'itinéraires de pratiques.

\subsection{Le glaneur, le bien-être au cœur de sa pratique}

Concrètement, le glaneur a une pratique assez superficielle, il se rend une fois par semaine en cours et n'introduit pas de pratique de yoga chez lui. Ses motivations restent d'abord centrées autour d'une pratique corporelle de bien-être associée ici aux sensations de détente et de calme. Ce glaneur peut, par périodes, souhaiter intensifier sa pratique, surtout lorsqu'il traverse une période plus difficile. Le yoga devient sa «pilule miracle », pour reprendre un terme d'un de mes interlocuteurs. Enfin, sa pratique du yoga a assez peu de répercussions, ou par petites touches, dans sa vie quotidienne.

Émilie pratique depuis trois ans, mais s'est interrompue au bout d'un an à cause de changements dans son emploi du temps. Elle n'a pas repris tout de suite, « se trouvant toujours des excuses ». Elle a commencé le yoga afin de se « recentrer », d'« être dans le moment présent », de " prendre soin d'elle ». Émilie a essayé de pratiquer chez elle, mais ne trouve ni le bon espace dans son appartement, ni le temps, ni la motivation. Elle en fait une semaine, puis arrête. Elle attend un " déclic », d'en « ressentir plus le besoin », " peut-être en vieillissant ». Lorsqu'elle me parle de sa pratique, elle dit avoir « honte », ne pas « être une bonne élève ». Elle note certains changements, notamment corporels. " Je suis en meilleure relation avec moi-même, savoir comment je fonctionne, je suis plus en paix avec moi-même, je connais aussi mieux mon corps. Et vis-à-vis des autres [...] j'ai l'impression qu'on ne peut plus m'agresser, d'être plus zen à l'intérieur. » (Émilie, pratiquante, 41 ans, Suisse)

Comme Émilie, le glaneur picore certains éléments qui l'intéressent et rejette les contraintes liées à une pratique régulière. Il comprend le yoga d'abord comme une pratique de bien-être. Ce bien-être renvoie au fait de se « sentir détendu » ou « d'être dans le moment présent ». Le glaneur n'a pas pour objectif d'explorer d'autres dimensions du yoga, ni la philosophie, ni les textes, ni la spiritualité, ni même de changer d'hygiène de vie. La finalité du yoga est une détente corporelle et mentale.

\subsection{Le bien-être, une porte d'entrée vers une écoute de soi pour les enthousiastes}

L'enthousiaste souhaite approfondir sa pratique. De nombreux parcours de vie évoquent ce passage. Cette curiosité émane de la prise de conscience des effets bénéfiques du yoga et résulte dans la capacité à s'astreindre à une régularité.

Katia pratique depuis trois ans et envisage de faire une formation de professeur. Elle a débuté avec son conjoint, tout d'abord pour trouver une activité sportive. « J'ai senti qu'il y avait un fort potentiel. Je dirais que c'était un champ ouvert sur plein de choses, 
un peu un nouveau stade, où tu rentres dans un autre monde. Peut-être quelque chose que j'attendais en fait, je [ne] dirais peut-être pas une révélation, mais il y a un peu de ça. » (Katia, pratiquante, 36 ans, Française). Pour Katia, le yoga est une "globalité » qui comporte des dimensions spirituelle, physique, et elle le définit comme une «maîtrise du corps pour calmer l'esprit, les émotions, afin d'atteindre des niveaux plus subtils de conscience de soi ». Katia pratique chez elle, avec ou sans son conjoint, suit toujours le même professeur de yoga, avec qui elle est déjà partie faire trois retraites, une par an. Depuis qu'elle a commencé le yoga, elle a découvert un véritable intérêt pour la nature, elle parle beaucoup de l'" union » avec le " grand tout », de ressentir de l'" énergie », notamment à travers les exercices de respiration. Parallèlement à sa pratique du yoga, Katia est en reconversion professionnelle pour aider les agriculteurs en conversion vers le biologique. Sa démarche n'est pas la conséquence de sa pratique du yoga, elle s'inscrit dans une volonté de " vivre plus en harmonie avec elle-même » et son environnement et de « donner un sens à son existence ».

Comme Katia, les enthousiastes décrivent de nombreux changements dans leur vie quotidienne, qui passent d'abord par des sensations corporelles : ressentir moins de tensions, de douleurs, de stress, d'être plus calme. Ils apprennent des techniques dans le yoga pour gérer leurs émotions au quotidien, respirer différemment dans des situations désagréables ou de stress. Souvent, ces premières modifications corporelles entraînent des transformations dans leurs rapports avec les autres. Beaucoup constatent qu'ils se mettent moins en colère, qu'ils écoutent plus, qu'ils sont plus heureux, ouverts ou bien qu'ils travaillent mieux, qu'ils sont plus efficaces, concentrés, etc.

D'autres dimensions peuvent être bouleversées sans que ces changements soient systématiques. Certains changent d'alimentation en réduisant quelques aliments (viande, alcool, tabac, café, thé, chocolat, etc.) ou deviennent végétariens (Nizard, 2020b). D'autres modifient leurs pratiques de santé en recourant plus aux médecines non médicamenteuses. Certains découvrent ou approfondissent des dimensions spirituelles (Nizard, 2020c). L'exemple de Katia montre qu'elle recherche à la fois à se sentir plus calme, tout en se maîtrisant et en approfondissant d'autres dimensions du yoga, comme la philosophie ou la spiritualité. Le bien-être, en plus d'être synonyme de détente, devient associé au sentiment d'une union holiste, entre le corps et l'esprit, voire d'une communion avec les autres, l'environnement, le «tout». Cette description du bien-être renvoie aux discours décrits par Puustinen dans les journaux spécialisés du yoga, alors que le bien-être réduit à la seule détente s'inscrit plutôt dans le discours de la presse féminine en général.

Ce passage du statut de glaneur à celui d'enthousiaste est parfois rapide. Certaines personnes parlent de quelques semaines, d'autres mettent des années. Étant donné l'homogénéité sociale (sexe, catégorie sociale) des pratiquants de yoga rencontrés, les raisons pour lesquelles les personnes s'impliquent plus ou moins dans le yoga relèvent plutôt de leur motivation première, de leurs attentes, de leur activité physique et de leur trajectoire de vie. Les personnes qui avaient une activité sportive avant de 
commencer le yoga se dirigent plutôt vers des courants de yoga assez dynamiques comme l'ashtanga, le vinyasa ou le hot yoga ${ }^{32}$.

Ensuite, certains facteurs favorisent ce passage. Premièrement, après la pratique de yoga, la personne ressent de tels bénéfices qu'elle souhaite explorer d'autres dimensions que les postures. Deuxièmement, les motivations pour débuter le yoga influencent l'implication. En effet, si cela survient après un deuil, un choc, une volonté profonde de changements, donc une «bifurcation » et que le yoga répond à leurs besoins, alors les pratiquants sont prêts à s'enthousiasmer. Ce facteur explique une adhésion plus rapide et plus profonde dans le yoga, qui n'existe pas dans les parcours de vie des glaneurs. On retrouve cet élément dans d'autres recherches comme dans le développement personnel. Nicolas Marquis (2014), qui s'est intéressé au marché des livres de développement personnel, parle de «brèche » biographique où les personnes cherchent « un sens » à leur vécu. Sur nos terrains, cette recherche de sens fait plutôt écho aux motivations des enthousiastes ou des passionnés. En effet, les glaneurs n'évoquent pas de changement dans leur situation matrimoniale, professionnelle ou de problèmes de santé. Au contraire, les enthousiastes ou les passionnés mentionnent très souvent des éléments de rupture qui les motivent pour rechercher un sens à ce qui s'est passé ou pour reprendre le « contrôle » sur une situation qui les dépasse.

Du coup, les enthousiastes souhaitent retrouver des sensations de « détente », parfois des « étirements », parfois « des moments dédiés à soi », « à l'écoute du corps ", « à apprendre à être dans le moment présent », « à répondre à un manque » ou « à un besoin ». Pour eux, le bien-être est d'abord la résultante d'une « écoute de son corps » et apparaît comme une porte d'entrée pour s'astreindre à une plus grande maîtrise de soi. Il ouvre la voie à une plus grande écoute des sensations corporelles, des émotions. Cette attention corporelle amène peu à peu l'enthousiaste à observer ses réactions, ses émotions. Pourtant, le yoga renvoie beaucoup plus à une « sorte d'idéal existentiel » tel que le décrit Garnoussi (2011, 2018).

\subsection{Du bien-être à la maîtrise de soi, les passionnés}

« Je travaillais dans une grosse boîte, 13 et 16 heures par jour, un stress insoutenable. [...] J’ai commencé le yoga entre midi et deux. [...] Je voyais tous les changements que tu vois au début. Tu sors, t'es tellement bien. Tu relativises. Tu prends sur toi. Tu vois la bonté chez les gens. [...] Pendant deux ans, je m’entraînais tous les

\footnotetext{
32 Étant donné que les pratiquants peuvent suivre plusieurs cours de yoga et changer dans le temps, il n'a pas été possible de croiser des données entre courant de yoga et implication. Parmi les personnes rencontrées, celles qui n'avaient aucune activité physique avant le yoga semblent moins enclines à changer leur hygiène de vie (alimentation, régularité dans la pratique, etc.), donc à se passionner pour le yoga que celles qui ont eu ou sont des sportifs par ailleurs.
} 


\begin{abstract}
jours. Et puis, je suis arrivée à un stade où mon yoga n'évoluait pas comme j'avais envie, parce que mon travail prenait encore trop de place. J'ai pris une année sabbatique et j'ai fait mon teacher training. [...] Là, ça a beaucoup changé parce que le yoga avait un aspect dévotionnel. Ce n'était pas pour faire du yoga et me sentir bien. [...] Tu vas aussi profond que tu peux aller. [...] Maintenant, je me réveille à $5 \mathrm{~h}$, parce que je donne des cours à $6 \mathrm{~h} 30$. [...] Je me lève, je me douche. Je médite, je fais des exercices respiratoires, puis les postures. [...] Ça oscille entre une heure et trois heures et demie, à jeun. Je mange deux repas par jour. Je fais un grand petit déjeuner et mange en fin de journée.» (Amandine, professeure, 30 ans, Suisse)
\end{abstract}

Encore plus loin dans l'implication, pour les passionnés, le yoga est un mode de vie, il est devenu « indispensable ». Ils décrivent le yoga comme un « besoin », un « virus », une " révélation », une " quête de soi ». L'exemple d'Amandine montre comment elle a quitté son travail, approfondi sa pratique, puis fait du yoga son quotidien, son hygiène de vie.

L'adhésion peut être immédiate ou venir avec le temps. Les personnes qui s'engagent plus profondément dans le yoga sont souvent celles qui ont connu une bifurcation claire, rapide voire brutale, comme une maladie, un divorce, un deuil d'une personne proche. Ces événements sont décrits par les pratiquants comme des «sonnettes d’alarme », des événements parfois traumatiques qui remettent profondément leur vie en question.

Souvent, les passionnés ont déjà fait de nombreux stages, ont effectué plusieurs retraites. Ils ont un discours élaboré sur leur corps, sur la place du yoga dans leur vie et démontrent combien le yoga les a transformés en touchant tous les domaines (corporels, émotionnels, professionnels, sociaux, spirituels). Comme Amandine, de nombreux passionnés ont fait une formation pour devenir professeur, d'abord pour approfondir leur pratique ou pour explorer de nouveaux aspects. Ils ont alors lu les textes du yoga perçus comme canoniques (les Yoga-Sutra, la Bhagavadgita). Les passionnés emploient des termes sanskrits lorsqu'ils parlent du yoga, au minimum pour nommer les postures. Certains sont allés en Inde ou ont développé un intérêt pour l'hindouisme. Quelques personnes exercent d'autres activités connexes. Mahé a repris des études de sanskrit, Dorothée suit un séminaire d'histoire de l'art, notamment indien à l'école du Louvre, Chiara fait de la danse indienne, Lola suit des cours de chant de mantras. Plusieurs ont fait des séjours à l'étranger pour pratiquer le yoga, souvent en Inde, mais pas systématiquement. Concernant les voyages et les loisirs, ils assistent à des conférences, partent faire un stage de yoga au moins une fois par an.

Ces personnes expriment le désir d'appartenir à une communauté afin de partager cette pratique avec d'autres personnes qui la comprennent. Ce besoin émane souvent d'un mode de vie ascétique (régularité dans les exercices corporels, adoption d'un régime végétarien, méditation, changements de relations sociales, loisirs dédiés 
quasiment exclusivement au yoga), parfois peu compris par l'entourage ou les autres personnes qui ne pratiquent pas le yoga. Le yoga est une hygiène de vie quotidienne (Nizard, 2020c). Les passionnés pratiquent au moins quatre fois par semaine, si ce n'est tous les jours. Le yoga n'est plus une activité de détente, c'est un projet de vie. Les passionnés comme les enthousiastes adhèrent aussi à un système de valeurs incorporées et communes au groupe qui animent leurs actions, ce qui définit un « ethos » (Droz, 2014). Ils évoquent souvent « l'esprit du yoga » qui se réfère à des règles morales (honnêteté, humilité, compassion, humanité, bienveillance, respect, tolérance), de comportements (être végétarien, respecter une discipline, une hygiène de vie avec une pratique quotidienne des postures, de la méditation). À ces valeurs morales s'ajoutent certains principes : le yoga peut être pratiqué par tous, quels que soient son sexe, son âge, son origine sociale, ses capacités physiques et l'expérience corporelle supplante la compréhension intellectuelle.

Chez les passionnés, le discours autour du bien-être se fait rare, voire il est perçu négativement. Pour eux, le bien-être ternit le yoga, qui ne se limite pas à cette dimension.

« Ça m'énerve tout ce marché autour du yoga bien-être. Ah oui, ces profs., ils ont compris comment faire du business, mais ils n'ont rien, mais alors rien compris au yoga. L'apaisement du corps et du mental, c'est juste une étape. Il faut maintenir le corps en bonne santé, pour accéder à la transcendance. Le but du yoga, c'est bien l'union. » (Mahé, 42 ans, professeur français)

Ce discours de Mahé fait écho à celui d'Amandine (supra), ils illustrent la tension existant autour de ces représentations du yoga associé au bien-être. Ici, la santé et non la détente est une étape. Mahé se réfère ici aux textes canoniques du yoga, comme les Yoga-Sutra, où les postures sont l'une des huit étapes du yoga. Ces textes décrivent, en effet, que le yogi, doit garder une hygiène de vie exemplaire (morale, physique et de purification corporelle), pour ensuite, pouvoir accéder à d'autres niveaux de méditation, puis atteindre la libération du cycle des réincarnations. Aussi, la finalité de la pratique du yoga n'est pas une détente physique, mais bien une "quête de soi », un idéal existentiel, de santé et/ou une spiritualité, dimensions valorisées qui supplantent la simple détente.

\section{Compréhension sociohistorique du bien-être}

Les évolutions du yoga et du bien-être illustrent des transformations sociales. Pour certains sociologues, cette « injonction au bien-être » (Heilbrunn, 2019) est représentative d'une responsabilisation croissante des individus afin qu'ils agissent sur eux-mêmes, qu'ils assument les conséquences de leurs actes et maximisent leurs potentiels (Cabanas, Illouz, 2018). Dans cette obligation du bien-être, le discours reste suffisamment diversifié pour permettre à chaque individu de se reconnaître et de partir à la « conquête 
de lui-même» (Ehrenberg, 1991). Si les sujets ressentent du stress, de l'anxiété, c'est parce qu'ils ne se seraient pas donné les moyens de réussir (Cabanas, Illouz, 2018).

Ehrenberg (2011) souligne que dans les années 1970, le nombre de thérapies corporelles parfois qualifiées "d'alternatives » a explosé. En mettant le corps au centre, toutes ces thérapies donnent une place croissante à la capacité d'action et de choix de l'individu. Aujourd'hui, ce phénomène s'est amplifié. La responsabilité de la santé et du bien-être n'est plus sociale, mais individuelle. Elle met l'accent sur la capacité de l'individu à agir et change la place de l'individu dans la société. Pour Ehrenberg, la question du bien-être illustre des transformations dans les idéaux sociaux, les représentations collectives. L'autonomie individuelle devient la valeur la plus importante. En effet, la santé, le bien-être s'illustrent par l'autodiscipline, par la capacité de l'individu à démontrer qu'il est maître de son corps et de ses actions ${ }^{33}$.

Si Cabanas et Illouz (2018) analysent cette absence de bien-être ou de réussite comme un signe d'une approche déficitaire de l'individu, pour Ehrenberg (2011), le paradigme aurait changé puisqu'aujourd'hui il s'agirait plutôt d'une approche en termes de potentialité, d'un « être capacitaire» (Ehrenberg, 2011) ou d'un individu « décideur 》 (Garnoussi, 2011), c'est-à-dire que la société imposerait à l'individu d'agir sur lui-même. Ainsi, ces idéaux d'autonomie et de bien-être se reconfigurent eux-mêmes. L'expression du bien-être comme activité individuelle, souvent analysé comme un repli sur soi, peut se lire comme une incorporation ${ }^{34}$, une autonomisation de cette injonction au bienêtre, laquelle se base sur le fait que l'individu répondrait à « une demande de transformation personnelle par un travail sur l'intériorité » (Garnoussi, 2011).

Le contexte sociohistorique décrit par ces auteurs explique à nos yeux l'engouement croissant pour le yoga aujourd'hui. Pourtant, il touche un public très spécifique, comme nous l'avons vu, les femmes, les urbains et les personnes appartenant à des catégories socioprofessionnelles moyennes ou supérieures. Les travaux de Eeva Sointu (2006, 2008), Linda Woodhead $(2007,2008)$ ou Carine Plancke (2020) apportent des arguments pertinents pour expliquer cette surreprésentation féminine. Pour ces auteures, les femmes européennes du XXI siècle sont enchâssées dans deux modes identitaires : l'un féminisé, qui repose sur l'impératif de devoir prendre soin des autres; l'autre masculinisé, reposant sur l'impératif de devoir de prendre soin de soi. Or, comme sur nos terrains, ces femmes travaillent majoritairement dans le social ou le médico-social, donc dans des métiers qui se dédient à autrui. Ainsi, les pratiques telles

\footnotetext{
${ }^{33}$ Propos recueillis lors de la conférence « Bien-être, santé mentale, autonomie » donnée par Ehrenberg lors du colloque Bien-être au Nord et au Sud : explorations, contradictions, pouvoir et pratiques du 22 au 25 octobre 2019, organisé par Sciences Po de l'Université de Bordeaux. ${ }^{34}$ Cette question de l'incorporation a été approfondie autour des questions d'apprentissage et du rôle du professeur dans cette interprétation du bien-être (Nizard, 2020a). Le mécanisme de l'affinement de l'attention corporelle et notamment de l'appréhension de la proprioception a fait l'objet d'un chapitre dans l'ouvrage sur la place des sens et des sensations dans les apprentissages (Nizard, à paraître).
} 
que le yoga (Plancke, 2020) ou les « spiritualités holistes » (Sointu, Woodhead 2008) qui mettent l'accent sur l'épanouissement personnel, permettraient à ces femmes de valoriser leur autonomie, leur épanouissement personnel, caractères plutôt « masculinisés » (Sointu, 2006). Ces pratiques aideraient à revendiquer (Plancke, 2020) ou à conjuguer ces deux modes identitaires (Sointu, Woodhead, 2008).

L'analyse des discours tenus par les pratiquants de yoga montre en effet une adhésion à l'idée qu'ils seraient capables d'agir sur eux-mêmes, pour accéder au bien-être (glaneurs), pour développer de nouvelles compétences (enthousiastes) ou pour se transformer (passionnés). L'autonomie, la volonté d'agir sur soi, l’importance donnée à l'apprentissage renvoient à ces transformations de soi caractéristiques des sociétés modernes et mises en exergue par Ehrenberg.

Comme Garnoussi (2018) le montre sur d'autres terrains, la centralité des références $\mathrm{au}$ bien-être permet de mettre en évidence des mutations des croyances contemporaines. Sans percevoir le yoga comme une activité de bien-être, l'abnégation dans la pratique, le fait de faire du yoga un projet de vie démontrent une volonté de maîtrise de soi, de prendre possession de son corps. Il s'agit bien ici d'un «idéal existentiel pragmatique et utilitariste » et l'argument légitimant est bien de donner la prévalence à «l'expérience intérieure» que chacun peut (voire devrait) cultiver (Garnoussi, 2018). Pour autant, ces exemples montrent une intériorisation variable de ces injonctions sociales. En effet, plus les pratiquants de yoga s'autonomisent, moins ils se réfèrent au bien-être. Plus les enthousiastes, puis les passionnés, incorporent ce devoir de prendre soin d'eux-mêmes, plus ils se contraignent et se détachent de la recherche de détente. Ici, le yoga peut se lire comme un droit au bien-être pour les glaneurs, un devoir de prendre soin de soi pour les enthousiastes et une hygiène de vie contraignante pour les passionnés. Les enthousiastes et surtout les passionnés adhèrent alors à un ensemble de valeurs, de comportements qui donnent une assise à cette recherche de santé et/ou de spiritualité.

\section{Conclusion}

Historiquement et socialement, le yoga s'est profondément transformé et peut aujourd'hui être compris comme une méthode psychocorporelle donnant la prévalence au bien-être, à la santé et/ou à la spiritualité (Nizard, 2020d). Si le yoga est associé au bien-être dans les représentations médiatiques, en réalité il cache une diversité de pratiques. Le bien-être est synonyme de « détente», s'oppose au stress pour les glaneurs, alors qu'il apparaît comme la résultante d'une écoute approfondie du corps, des sensations pour les enthousiastes. Les passionnés rejettent généralement cette association entre yoga et bien-être, d'une part parce que le bien-être renvoie à une représentation d'un yoga plus commercial (avec des formations courtes, une méconnaissance d'autres dimensions du yoga comme les textes, la philosophie, la spiritualité), et d'autre part, parce que le yoga est devenu pour eux un véritable mode de vie. Il n'est plus un loisir 
de détente, mais implique régularité, restrictions (alimentaires, de rythme de vie, sociales, etc.).

À la lumière des recherches des sociologues sur le bien-être, il est possible de comprendre ce phénomène comme significatif d'une responsabilisation croissante des individus afin qu'ils prennent soin de leur corps et d'une démonstration d'une capacité à mieux se maîtriser. L'exemple des pratiquants de yoga montre aussi que cette expérience intérieure, initiée parfois par le bien-être, entraîne des transformations des croyances sur le corps, sur son rapport à soi et au monde. Ce contexte social permet plus généralement d'expliquer pourquoi le yoga est associé tantôt au bien-être, tantôt à une spiritualité tantôt à une méthode thérapeutique. Ces trois registres ne sont pas antithétiques. D'autres itinéraires de pratiques soulignent un intérêt plus marqué pour la santé et/ou pour la spiritualité. Dans le discours des pratiquants rencontrés, il peut y avoir une juxtaposition des arguments. Certains glaneurs valorisent le bien-être tout en percevant une spiritualité dans leur pratique, alors que certains passionnés se détachent totalement des dimensions spirituelles dominantes dans certains courants ( $\mathrm{Si}$ vananda, Jivamukthi ou Kundalini). Les courants de yoga portés plus sur le physique, la santé et/ou la spiritualité mobilisent la même rhétorique autour de la responsabilité individuelle, de la prise de conscience de soi, de la capacité à se transformer. Ici, l'accent a été mis sur le bien-être dans les pratiques du yoga comme modalité pour s'impliquer dans la pratique et ouvrir vers une nouvelle transformation des modes de vie.

\section{Bibliographie}

AleXandrova A. (2017), A Philosophy for the Science of Well-Being, New York, Oxford University Press.

Ali-KNiGHT J., EnSOR J. (2017), « Salute to the sun: An exploration of UK Yoga tourist profiles », Tourism Recreation Research, vol. 42, n 4, p. 484-497.

Altglas V. (2005), Le Nouvel Hindouisme occidental, Paris, CNRS Éditions, (« Sociologie »). Alter J. (2004), Yoga in modern India, the body between science and philosophy, Princeton/Oxford, Princeton University Press.

Bhalla N., Moscowitz L. (2019), "Yoge for Every(body)? A Critical Analysis of the Evolution of Yoga Representation across Four Decades in Yoga Journal », Journal of Magazine Media, vol. 19, n 1, p. 75-103.

BERNARD M. (1976), Le Corps, Paris, Jean-Pierre Delarge.

BROWN K. W., RYAN R. M. (2003), « The benefits of being present: Mindfulness and its role in psychological well-being. », Journal of Personality and Social Psychology, vol. 84, $\mathrm{n}^{\circ} 4$, p. 822-848. En ligne, consulté le 19 juin 2019. URL: https://selfdeterminationtheory.org/SDT/documents/2003_BrownRyan.pdf.

CABANAS E., IlLouz E. (2018), Happycratie. Comment l'industrie du bonheur a pris le contrôle de nos vies, Paris, Premier Parallèle.

CAMERON O. (2001), «Interoception: the inside story - a model for psychosomatic 
processes", Psychosomatic Medicine, vol.63, p.697-710. En ligne, consulté le 04 septembre 2019. URL: https://insights.ovid.com/crossref?an=00006842200109000-00001

Ceccomori S. (2001), Cent Ans de Yoga en France, Paris, Edidit.

CEDerström C., Spicer A. (2016), Le syndrome du bien-être, Paris, Éditions l’Échappée.

CoRsín Jimenèz A. (dir.) (2008), Culture and Well-Being: Anthropological Approaches to Freedom and Political Ethics, Londres, Pluto Press.

De Michelis E. (2005), A History of Modern Yoga, Patañjali and Western Esotericism, Londres/New York, Bloomsbury Academic.

DeSPONDS MEYLAN S. (2007), L'enseignement de yoga européen entre adhikāra et pédagogie. Une analyse de la qualification socio-religieuse des enseignants dans la rencontre entre l'Union européenne de yoga et le lignage de T. Krishnamacharya, thèse de doctorat, Lausanne, Faculté des lettres de l'université de Lausanne.

DinESH T. (2013), "Effect of 6 weeks of kapalabhati pranayama Training on Peak Expiratory Flow », Scholars Academic Journal of Biosciences, vol. 1, n 4, p. 111-114. En ligne, consulté le 04 octobre 2015. URL : https://www.bibliomed.org/?mno=206687

Droz Y. (2014), Retours croisés des Afriques aux Amériques: de la mobilité religieuse, Paris, Karthala.

EHREnBerg A. (1991), Le culte à la performance, Paris, Hachette.

EHRENBERG A. (2011), « La société du malaise. Une présentation pour un dialogue entre clinique et sociologie », Adolescence, vol. 3, n 77, p. 553-570. En ligne, consulté le 27 novembre 2019. URL : https://www.cairn.info/revue-adolescence-2011-3-page553.htm?contenu=article.

EISENMANn C. (2019), Spirituality as Social Practice - On the Construction of Reality in Yoga, Berlin, De Gruyter.

FORSÉ M., LANGLOIS S. (dir.) (2014), « Sociologie du bien-être », L’année sociologique, vol. 64, $\mathrm{n}^{\circ} 2$, p. 261-271.

FouCAult M. (1984), Histoire de la sexualité, T.III, Le souci de soi, Paris, Gallimard (« Tel »).

FREY B. (2011), « Subjective well-being, politics and political economy », Swiss Journal of Economics and Statistics, vol. 174, $\mathrm{n}^{\circ}$ 4, p. 397-415.

GARNOUSSI N. (2011), « Le Mindfulness ou la méditation pour la guérison et la croissance personnelle: des bricolages psychospirituels dans la médecine mentale», Sociologie, vol. 2, n³, p. 259-275. URL: https:/www.cairn.info/revue-sociologie2011-3-page-259.htm

GARNOUSSI N. (2018), «À la recherche du bien-être, déplacements et perspectives », in I. CoUTANT et S. WANG (dir.), Santé mentale et souffrance psychique : un objet pour les sciences sociales, Paris, CNRS Éditions, p. 227-245.

Grossetti M., Bessin M., BidART C. (dir.) (2009), Bifurcations. Les sciences sociales face aux ruptures et à l'événement, Paris, La Découverte.

Hauser B. (2013), Yoga Traveling: Bodily Practice in transcultural Perspective, New York, Cham. 
HÉAS S. (2020), « Les méthodes psychocorporelles aujourd'hui : de l'alternative douceur à une lenteur alternative?» in F. LEBRETON, C. Gibout (dir.), Vivre Slow. Enjeux et perspectives pour une transition corporelle, récréative et touristique, Nancy, Presses Universitaires de Nancy, p. 241-261.

HeILBRunN B. (2019), L'obsession du bien-être, Paris, Robert Laffont.

Hoyez A.-C. (2012), L'espace monde du yoga. De la santé aux paysages thérapeutiques mondialisés, Rennes, Presses Universitaires de Rennes.

Jerath R., Barnes V.A., Dillard-Wright D., Jerath S., Hamilton B. (2012), « Dynamic change of awareness during meditation techniques: neural and physiological correlates", Frontiers in human neuroscience, $\mathrm{n}^{\circ} 6$. En ligne, consulté le 18 octobre 2017.

URL :

https://www.frontiersin.org/articles/10.3389/fnhum.2012.00131/full

Lehto X. Y., Brown S., Chen Y., Alaistair M. (2006), « Yoga Tourism as a Niche Within the Wellness Tourism Market », Tourism Recreation Research, vol. 31, n 1, p. 25-35.

Mallinson J., Singleton M. (2017), Roots of Yoga, Londres, Penguin Books.

MARQUis N. (2014), Du bien-être au marché du malaise. La société du développement personnel. Paris, Presses Universitaires de France.

Moco O. (2007), « Bien-être », Quaderni, vol. 63, n 1, p. 14-15.

MORI L., SQUARCINI F. (2008), Fra yoga, storia e mercato, Rome, Carocci.

NEwcombe S. (2005), "Spirituality and 'mystical religion' in contemporary society: a case study of British practitioners of the Iyengar method of yoga », Journal of Contemporary Religion, vol. 20, n 3, p. 305-322.

Nichter M. (2000), Fat Talk: What Girls and Their Parents Say about Dieting, Cambridge, Harvard University Press.

NiZARD C. (2019), Du souffle au corps. Apprentissage du yoga en France, en Suisse et en Inde, Paris, L'Harmattan.

NiZARD C. (2020a), « S’ac-corps-der, approfondissement de l'attention corporelle dans les pratiques du yoga », in B. ANDRIEU (dir.), De l'intérieur. Manuel d'Emersiologie, Milan, Éditions Mimesis, p. 272-340.

NizARD C. (2020b), « Végétarisme et yoga. Des représentations à la construction de soi », SociologieS, En ligne, consulté le 03 novembre 2020. URL: https://journals.openedition.org/sociologies/12618.

NiZARD C. (2020c), «En quête de soi », L'ethnographie, n 3-4. En ligne, consulté le 03 novembre 2020.URL :

https://revues.mshparisnord.fr/ethnographie/index.php?id=689

NizARD C. (2020d), «La "spiritualité" dans le yoga moderne: nouveau mouvement religieux, méthode de perfectionnement de soi ou désintérêt ? ", ¿ Interrogations? Revue pluridisciplinaire de sciences humaines et sociales, $\mathrm{n}^{\circ} 31$, à paraître.

NizARD C. (à paraître), « Conférer du sens à l'expérience sensible. Le cas du yoga », in J. CANDAU et V. BATTESTI (dir.), Anthropologie de l'apprentissage sensoriel : Apprendre par 
les sens, apprendre les sens, Paris, Éditions Pétra.

PAwiN R. (2014), « Le bien-être dans les sciences sociales : naissance et développement d'un champ de recherches ", L’Année sociologique, vol. 64, n² 2, p. 273-294.

PERRIN E. (1984), Cultes du corps. Enquête sur les nouvelles pratiques corporelles, Lausanne, Éditions Favre.

Plancke C. (2020), « Yoni Touch and Talk: Sacralizing the Female Sex through Tantra », Sexualities, vol. 23, n 5-6, p. 834-848.

Prakash S, Upadhyay-Dhungel K. (2005), « Chandra Anuloma Viloma pranayama modified cardiorespiratory functions", Janaki Medical College Journal of Medical Sciences, vol. 3, $n^{\circ} 1$, p. 45-51.

PRICE C. J., Hooven C. (2018), « Interoceptive Awareness Skills for Emotion Regulation: Theory and Approach of Mindful Awareness in Body-Oriented Therapy (MABT)», Frontiers in psychology, vol. 9, $\mathrm{n}^{\circ}$ 798. En ligne, consulté le 04 septembre 2019. URL : https://www.frontiersin.org/articles/10.3389/fpsyg.2018.00798/full.

PUUSTINEN L. K, RAUTANIEMI M. (2015), « Wellbeing for sale: representations of yoga in commercial media », Temenos: studies in comparative religion presented by scholars in Denmark, Finland, Norway and Sweden, vol. 51, $n^{\circ} 1$, p. 45-70.

RuIz G. (2010), « Le yoga, un business en lévitation », L’hebdo, En ligne, consulté le12 avril 2018. URL : https://genevieveruiz.com/en/le-yoga-un-business-en-levitation/

SARASWATi N. (2009), Prana and pranayama, Munger/Bihar, Yoga Publications.

Schulte P. A., Guerin R. J., Schill A. L., Bhattacharya A. (2015), «Considerations for Incorporating "Well-Being" in Public Policy for Workers and Workplaces", Prompting Public Health Research, Policy, Practice and Education, vol. 105, n 8, p. 3144.

Seligman M. (2013), S'épanouir: pour un nouvel art du bonheur et du bien-être, Paris, Belfond.

SinglEton M. (2010), Yoga Body. The origins of modern posture practice, New York, Oxford University Press.

SoINTU E., WoodHEAD L. (2008), « Spirituality, Gender and Expressive Selfhood », Journal for the Scientifif Study of Religion, vol. 47, n² 2, p. 259-276.

SoINTU E. (2006), « The Search for Wellbeing in Alternative and Complementary Health Practices ", Sociology of Health and Illness, vol. 28, n 3, p. 330-349.

StRAuss S. (2005), Positioning Yoga: balancing acts across cultures, Oxford/New York, Berg Publishers.

TARDAN-MASQUELIER Y. (2002), «La réinvention du yoga par l'Occident », Études, n 396, p. 39-50.

Weber M. (1992), Essais sur la théorie de la science, Paris, Pocket.

WoodHead L. (2007), "Why so Many Women in Holistic Spirituality? A Puzzle Revisited », in K. Flanagan, P. Jupp (dir.), The Sociology of Spirituality, Aldershot, Ashgate, p. 115-125. 
White D. G. (2012), Yoga a Brief History of an Idea, Princeton, Princeton University Press.

\section{Annexe : caractéristiques des terrains}

Mori et Squarcini (2008) ont recensé vingt-six courants de yoga, mais depuis, de nombreux mélanges hybrides sont apparus avec par exemple le yoga des femmes enceintes, pour les enfants, le yoga paddle, le dog-yoga... Il n'existe pas de statistiques en France sur le nombre de pratiquants par courant de yoga, d'autant que de nombreux professeurs ne se reconnaissent pas dans un courant spécifique. Lors de l'enquête, l’idée a été de rendre compte d'un panel de pratiques en choisissant des courants institutionnalisés (comme Sivananda), des professeurs s'inscrivant ouvertement dans un courant dont le discours dominant était plus porté sur la dimension spirituelle, sportive ou thérapeutique et enfin de rencontrer des enseignants créant des mélanges composites (la majorité aujourd'hui). Pour cela, j'ai contacté par email une trentaine de professeurs identifiés grâce à Internet. Une dizaine ont accepté de réaliser un entretien. Par la suite, j'ai assisté à leurs cours en tant que pratiquante. Dès le début, je me suis présentée aux élèves pour qu'ils connaissent mon identité et leur proposer de réaliser un entretien. De cette manière, une quarantaine d'entretiens ont été réalisés. Les autres personnes ont été rencontrées lors de retraites ou de festival.

\begin{tabular}{|c|c|c|c|c|c|}
\hline Pays & Terrains & Contexte & Durée & $\begin{array}{c}\text { Nombre } \\
\text { d'entretiens }\end{array}$ & $\begin{array}{c}\text { Nombre approximatif } \\
\text { d'heures d'observation/ } \\
\text { cours }\end{array}$ \\
\hline \multirow{4}{*}{ France } & Paris & Cours & $\begin{array}{c}\text { ponctuels, week } \\
\text { end entre } 2013 \\
\text { et } 2018\end{array}$ & \multirow{4}{*}{23} & 50 heures $/ 30$ cours \\
\hline & Orléans & $\begin{array}{c}\text { Retraite } \\
\text { ashram Siva- } \\
\text { nanda }\end{array}$ & $\begin{array}{c}3 \times 1 \text { semaine : } \\
2013,2014 \text { et } \\
2015\end{array}$ & & 210 heures $/ 150$ pratiques \\
\hline & Ardèche & $\begin{array}{c}\text { Retraite } \\
\text { ashram Do- } \\
\text { maine du } \\
\text { Taillé }\end{array}$ & $\begin{array}{l}10 \text { jours } \\
\text { juin } 2015\end{array}$ & & 80 heures $/ 50$ pratiques \\
\hline & Montpellier & Cours & 2013 & & 80 heures $/ 50$ pratiques \\
\hline
\end{tabular}




\begin{tabular}{|c|c|c|c|c|c|}
\hline \multirow{4}{*}{ Suisse } & Gruyères & $\begin{array}{l}\text { Cours auprès } \\
\text { de trois pro- } \\
\text { fesseurs }\end{array}$ & 2013 à 2018 & \multirow{4}{*}{22} & 725 heures / 250 cours \\
\hline & Lausanne & $\begin{array}{l}\text { Cours ponc- } \\
\text { tuels }\end{array}$ & 2015 à 2018 & & 50 heures/30 cours \\
\hline & Fribourg & $\begin{array}{l}\text { Cours auprès } \\
\text { d'un profes- } \\
\text { seur }\end{array}$ & 2014 à 2018 & & 260 heures $/ 200$ cours \\
\hline & Zinal & $\begin{array}{c}\text { Congrès an- } \\
\text { nuel }\end{array}$ & $\begin{array}{l}\text { 2x1 semaine : } \\
2016 \text { et } 2017\end{array}$ & & 120 heures $/ 80$ cours \\
\hline \multirow{6}{*}{ Inde } & Bangalore & $\begin{array}{c}\text { Cours, Retraite } \\
\text { centre Vyasa }\end{array}$ & 1 mois en 2013 & \multirow{6}{*}{15} & 280 heures/190 pratiques \\
\hline & Neyyar Dam & $\begin{array}{c}\text { Retraite } \\
\text { ashram Siva- } \\
\text { nanda }\end{array}$ & $\begin{array}{l}2 \text { semaines en } \\
2013\end{array}$ & & 120 heures $/ 80$ cours \\
\hline & Mysore & Cours & 1 mois en 2013 & & 280 heures/190 pratiques \\
\hline & Chennai & Cours & 1 mois en 2013 & & 280 heures/190 pratiques \\
\hline & Dehli & $\begin{array}{c}\text { Cours, Retraite } \\
\text { dans l’Uttar } \\
\text { Pradesh }\end{array}$ & 2 mois en 2017 & & 560 heures $/ 280$ cours \\
\hline & Rishikesh & $\begin{array}{c}\text { Retraite } \\
\text { Ashram Par- } \\
\text { math Niketan }\end{array}$ & 1 mois en 2017 & & 120 heures $/ 80$ cours \\
\hline \multirow{2}{*}{ Autre } & Varsovie & $\begin{array}{l}\text { Congrès scien- } \\
\text { tifique }\end{array}$ & $\begin{array}{c}1 \text { semaine en } \\
2016\end{array}$ & \multirow{2}{*}{0} & \\
\hline & Londres & $\begin{array}{l}\text { Congrès scien- } \\
\text { tifique }\end{array}$ & $\begin{array}{c}1 \text { semaine en } \\
2017\end{array}$ & & \\
\hline TOTAL & & & & 60 & 3000 heures/1850 pratiques \\
\hline
\end{tabular}

